\title{
Elusive vector glueball
}

\author{
Mahiko Suzuki \\ Department of Physics and Lawrence Berkeley National Laboratory \\ University of California, Berkeley, California 94720
}

(November 1, 2018)

If the vector glueball $\mathcal{O}$ exists in the mass range that theory suggests, its resonant production cross section can be detected in $e^{+} e^{-}$annihilation only if the decay width is very narrow $(\leq$ a few $\mathrm{MeV})$. Otherwise $\mathcal{O}$ will be observed only indirectly through its mixing with $\psi^{\prime}$. We propose a few tests of the $\mathcal{O}-\psi^{\prime}$ mixing for future charm factories.

PACS number(s): 12.39.Mk, 13.20.Gd, 13.65.+i, 14.40.Gx

\section{INTRODUCTION}

While theorists expect that the glueballs exist as the flavorless hadrons made of gluons alone, the predicted masses vary from lattice QCD [1] to phenomenological models such as the bag-type model [2] and a naive constituent model [3]. Experiment provided a few candidates for the ground-state glueball of $J^{P C}=0^{++}$in the past, but no consensus has been reached. The glueball $\mathcal{O}$ of $1^{--}$is made of three gluons in the constituent picture so that its mass is expected to be roughly $50 \%$ higher than the lowest glueball mass. The recent lattice QCD calculation [1] predicts the mass of $\mathcal{O}$ at $3850 \pm 50 \pm 190$ $\mathrm{MeV}$ in the quenched approximation. It is amusing that this value happens to be very close to the $\psi^{\prime}$ mass (3686 $\mathrm{MeV})$. In contrast to the masses, very little is known even theoretically about the widths since they are determined by how the glueballs couple to light quarks.

A few puzzles exist in the hadronic $\psi^{\prime}$ decay. One is the so-called $\rho \pi$ puzzle, i.e., the severe suppression of $\psi^{\prime} \rightarrow \rho \pi$ 近. If both $J / \psi$ and $\psi^{\prime}$ decay into hadrons through the perturbative $g g g$ from the ${ }^{3} S_{1}$ states of $c \bar{c}$, we expect that their hadronic decays should be very similar to each other. Contrary to this expectation, the branching fractions are very different not only for $\rho \pi$ but also for many other exclusive hadron channels [5]. Since $\mathrm{B}(J / \psi \rightarrow \rho \pi) / \mathrm{B}(J / \psi \rightarrow \omega \pi)$ is close to $\mathrm{B}(J / \psi \rightarrow$ $g g g \rightarrow$ hadrons $) / \mathrm{B}\left(J / \psi \rightarrow \gamma^{*} \rightarrow\right.$ hadrons $)$ [6], the decay $J / \psi \rightarrow \rho \pi$ is just as strong as the naive expectation. The experimental fact that $\mathrm{B}\left(\psi^{\prime} \rightarrow \omega \pi\right) / \mathrm{B}(J / \psi \rightarrow$ $\omega \pi) \approx \mathrm{B}\left(\psi^{\prime} \rightarrow \ell^{+} \ell^{-}\right) / \mathrm{B}\left(J / \psi \rightarrow \ell^{+} \ell^{-}\right)$assures us that there is nothing anomalous about the $1^{-} 0^{-}$modes in the one-photon annihilation. There appears to be no perturbative $1^{-} 0^{-}$suppression that was once suggested as a resolution to the $\rho \pi$ puzzle [7]. Therefore the source of the $\rho \pi$ puzzle is in some property of $\psi^{\prime}$.

The other puzzle is an apparent excess of $\psi^{\prime} \rightarrow$ hadrons relative to $J / \psi \rightarrow$ hadrons [8,9]. To establish it quantitatively, we need more an accurate determination of the cascade decay branchings of $\psi^{\prime}$. The current data hint that there exists some unknown decay mechanism for $\psi^{\prime} \rightarrow$ hadrons other than the standard perturbative three-gluon decay. It is the $\psi^{\prime}-\mathcal{O}$ mixing that may resolve both puzzles by one shot \&1.7

In view of the precision charm experiment proposed at CESR and at Beijing, we pursue here the $\mathcal{O}-\psi^{\prime}$ mixing as quantitatively as possible. Assuming that the excess $\psi^{\prime} \rightarrow$ hadrons really exists and that it is caused by the $\mathcal{O}-\psi^{\prime}$ mixing, we estimate the resonant $\mathcal{O}$ production cross sections. We find that the $\mathcal{O}$ resonance peak will be detected in $e^{+} e^{-}$annihilation only if the decay width is very narrow $\left(\Gamma_{\mathcal{O}}<\right.$ a few $\left.\mathrm{MeV}\right)$. If the width is wider, we shall be able to find only indirect evidences for $\mathcal{O}$ through the $\psi^{\prime}$ decay. In a dedicated charmonium experiment, experimentalists should first confirm the excess in $\psi^{\prime} \rightarrow$ hadrons by determining more accurately the $\psi^{\prime} \rightarrow J / \psi$ cascade decay branchings, and then examine individual exclusive hadron channels of even G-parity such as $\omega \pi, \pi^{+} \pi^{-}$, and $\rho \eta$.

\section{II. $\mathcal{O}-\psi^{\prime}$ MIXING}

The $\mathcal{O}-\psi^{\prime}$ mixing is determined by the $2 \times 2$ mass matrix of $\mathcal{O}$ and $\psi^{\prime}$,

$$
\mathcal{M}=\left(\begin{array}{cc}
m_{\mathcal{O}}^{2}-i m_{\mathcal{O}} \Gamma_{\mathcal{O}} & f_{\mathcal{O} \psi^{\prime}} \\
f_{\mathcal{O} \psi^{\prime}} & m_{\psi^{\prime}}^{2}-i m_{\psi^{\prime}} \Gamma_{\psi^{\prime}}
\end{array}\right)
$$

Two eigenstates of $\mathcal{M}$ are the standard Breit-Wigner resonances. Since the complex mixing angle $\theta \simeq$ $\frac{1}{2} \tan ^{-1}\left[2 f_{\mathcal{O} \psi^{\prime}} /\left(\mathcal{M}_{11}-\mathcal{M}_{22}\right)\right]$ is very small in magnitude in the cases of our interest, we shall treat the mixing perturbatively and denote the physical BreitWigner states also by $\mathcal{O}$ and $\psi^{\prime}$ below. We shall discard $\left|m_{\psi^{\prime}}-m_{\mathcal{O}}\right| / m_{\psi^{\prime}} \ll 1$ wherever appropriate.

In addition to the standard perturbative $g g g$ decay, the physical $\psi^{\prime}$ state can decay through its mixing with $\mathcal{O}$ :

$$
\Gamma\left(\psi^{\prime} \rightarrow \mathcal{O} \rightarrow a l l\right)=\frac{\left|f_{\mathcal{O} \psi^{\prime}}\right|^{2}}{m_{\mathcal{O}}^{2} \Gamma_{\mathcal{O}}} F(\Delta m, \Gamma) .
$$

\footnotetext{
${ }^{1}$ The other idea is a large virtual $D \bar{D}$ configuration or a large ${ }^{3} D_{1}$ mixing in the $\psi^{\prime}$ state [10. All earlier attempts to resolve the $\rho \pi$ puzzle failed with the recent analysis of the $\psi^{\prime}$ decay modes, as was shown by Harris [0]. See also Ref. [11].
} 
where $F(\Delta m, \Gamma)$ comes from the $\mathcal{O}$ resonance shape;

$$
F(\Delta m, \Gamma)=\Gamma_{\mathcal{O}}^{2} /\left[4(\Delta m)^{2}+\Delta \Gamma_{\mathcal{O}}^{2}\right]
$$

with $\Delta m=\left|m_{\mathcal{O}}-m_{\psi^{\prime}}\right|$ and $\Delta \Gamma_{\mathcal{O}} \simeq \Gamma_{\mathcal{O}}\left(\gg \Gamma_{\psi^{\prime}}\right)$.

We parametrize the alleged excess in the hadronic $\psi^{\prime}$ decay as

$$
\Gamma\left(\psi^{\prime} \rightarrow \text { hadrons }\right) / \Gamma\left(\psi^{\prime} \rightarrow g g g\right)=1+\mathcal{E},
$$

where $\mathcal{E} \approx 0.6-0.7$ with large errors according to the current data [8,9]. This excess is attributed to the additional decay, $\psi^{\prime} \rightarrow \mathcal{O} \rightarrow$ hadrons. Since $\Gamma_{\mathcal{O}} \gg \Gamma_{\psi^{\prime}}$, even a tiny mixing can make this contribution as large as the perturbative $\Gamma\left(\psi^{\prime} \rightarrow g g g\right)$. Without knowing dynamics of $\mathcal{O}$ decay, let us assume for the moment that $\psi^{\prime} \rightarrow \mathcal{O} \rightarrow$ hadrons adds to $\psi^{\prime} \rightarrow$ ggg $\rightarrow$ hadrons in the total rate. That is, we assume no interference in the inclusive decay rate, though the interference is crucial in individual exclusive rates. This is not an absurd assumption: If the $g g g$ state of $\mathcal{O}$ differs substantially from the perturbative $g g g$ configuration of $\psi^{\prime}$ decay, it is capable of producing very different interference patterns for one exclusive channel to another, leading to suppression or enhancement of the exclusive rates, yet the interference terms cancel out in the inclusive rate. On this assumption, $f_{\mathcal{O} \psi^{\prime}}$ is constrained by the excess $\mathcal{E}$ as

$$
\left|f_{\mathcal{O} \psi^{\prime}}\right|^{2}=\frac{(25 \mathrm{MeV})^{4} \mathcal{E}}{F(\Delta m, \Gamma)} \times\left(\frac{\Gamma_{\mathcal{O}}}{1 \mathrm{MeV}}\right) .
$$

The $\psi^{\prime} \rightarrow \gamma^{*}$ transition strength defined by $f_{\gamma \psi^{\prime}} A^{\mu} \psi_{\mu}^{\prime}$ can be expressed as $f_{\gamma \psi^{\prime}}^{2}=\left(3 m_{\psi^{\prime}}^{3} / \alpha\right) \Gamma\left(\psi^{\prime} \rightarrow e^{+} e^{-}\right)$.

If $\mathcal{O}$ decays mainly as $\mathcal{O} \rightarrow q \bar{q} \rightarrow$ hadrons, and if the couplings of $\mathcal{O}$ to quarks are flavor blind, we could derive an additional interesting constraint with Eq. (5) by expressing $f_{\mathcal{O} \psi^{\prime}}$ with the $\mathcal{O} c \bar{c}$ coupling, and the $\mathcal{O}_{q \bar{q}}$ coupling with $\Gamma_{\mathcal{O}}$. The result is $\Delta m / \Gamma_{\mathcal{O}} \simeq 30 / \sqrt{\mathcal{E}}$.

\section{RESONANT PRODUCTION CROSS SECTIONS}

$$
\text { A. } e^{+} e^{-} \rightarrow \mathcal{O} \rightarrow \text { all }
$$

The glueball $\mathcal{O}$ is most favorably reached through $e^{+} e^{-} \rightarrow \gamma^{*} \rightarrow \psi^{\prime} \rightarrow \mathcal{O} \rightarrow$ all. The cross section at the $\mathcal{O}$ resonance peak is given by

$$
\sigma\left(e^{+} e^{-} \rightarrow \mathcal{O} \rightarrow a l l\right)=\frac{\pi \alpha\left|f_{\gamma \psi^{\prime}} f_{\mathcal{O} \psi^{\prime}}\right|^{2}}{m_{\mathcal{O}}^{7}(\Delta m)^{2} \Gamma_{\mathcal{O}}}
$$

In the unit of $\sigma\left(e^{+} e^{-} \rightarrow \mu^{+} \mu^{-}\right)$, the peak value is

$$
R=\frac{9\left|f_{\mathcal{O} \psi^{\prime}}\right|^{2} \Gamma\left(\psi^{\prime} \rightarrow \ell^{+} \ell^{-}\right)}{4 \alpha^{2}(\Delta m)^{2} m_{\mathcal{O}}^{2} \Gamma_{\mathcal{O}}} .
$$

The right-hand side is sizable only if the width $\Gamma_{\mathcal{O}}$ is very narrow and $\left|f_{\mathcal{O} \psi^{\prime}}\right|^{2} / \Gamma_{\mathcal{O}}$ is strongly enhanced by small
$F(\Delta m, \Gamma)$. (See Eq. ([).) For $\Gamma_{\mathcal{O}} \ll \Delta m, R$ is insensitive to $\Delta m$ and scales approximately as $1 / \Gamma_{\mathcal{O}}^{2}$;

$$
R \simeq\left(\frac{3.2 \mathrm{MeV}}{\Gamma_{\mathcal{O}}}\right)^{2} \mathcal{E}
$$

If $\mathcal{O}$ decays with three gluons falling apart into quarks by long-distance interactions, such a narrow width is unlikely. On the other hand, if short-distance physics suppresses conversion of $g g g$ into light quarks by $\alpha_{s}^{3}$, a very narrow width is not totally out of question. Note that $\Gamma=0.087 \mathrm{MeV}$ for $J / \psi$ by $\alpha_{s}^{3}$ suppression. Since we have no knowledge of how the glueballs decay, there is no orthodoxy about their widths.

Suppose that one finds a candidate of the $\mathcal{O}$ peak by scanning over the relevant energy range. If it is really the $\mathcal{O}$ resonance, the lepton-pair production cross section remains the same at the peak as at the side bands:

$$
R_{\ell^{+} \ell^{-}}=1, \quad(\text { peak }),
$$

since $\mathcal{O} \rightarrow q \bar{q} \rightarrow \gamma^{*} \rightarrow \ell^{+} \ell^{-}$is negligible. Another test is whether the increase in hadron events occurs all in $I=0$ or not. This is most easily checked by $G$-parity of the final states. At the continuum off the resonance, hadrons are produced through light quarks in $I=0$ and $I=1$ channels in the ratio of

$$
R_{I=0} / R_{I=1}=1 / 3, \text { (continuum). }
$$

That is, if one counts the final states of even pions and of odd pions at the continuum, the even-pion events dominate over the odd-pion events. This odd-to-even pion ratio should suddenly jump at the $\mathcal{O}$ resonance. Narrowness of width rules out light-quark resonances of $I=0$.

We comment on production of $\mathcal{O}$ through the light quarks instead of $\psi^{\prime}: e^{+} e^{-} \rightarrow q \bar{q}(q=u, d, s) \rightarrow \mathcal{O} \rightarrow$ hadrons. This production process is actually flavor$\mathrm{SU}(3)$-forbidden since the electromagnetic current of the $u, d, s$ quarks is an octet while $\mathcal{O}$ is a singlet. Production of $\mathcal{O}$ occurs only by $\mathrm{SU}(3)$ breakings. We can compute these amplitudes perturbatively, if we keep only the onshell contribution of $q \bar{q}$. The result is independent of $\Gamma_{\mathcal{O}}$ if $\Gamma_{\mathcal{O}}$ is given by the process $\mathcal{O} \rightarrow q \bar{q} \rightarrow$ hadrons:

$$
R=\frac{1}{48}\left(\frac{2 m_{s}}{m_{\mathcal{O}}}\right)^{4},
$$

where $m_{s}$ is the $s$-quark mass that enters through the quark mass splitting of the intermediate states. Even if $\mathcal{O}$ is produced through $s \bar{s}$ alone or through $u \bar{u}+d \bar{d}$ by the maximal $\mathrm{SU}(3)$ violation, the value of $R$ would be only 0.037. The off-shell $q \bar{q}$ contribution depends on the form factor damping of the $\mathcal{O} q \bar{q}$ vertex, which is expected to be soft since $\mathcal{O}$ is an extended object. Therefore the off-shell contribution cannot be much larger than the onshell contribution. We may safely dismiss the $\mathcal{O}$ production through light quarks. 


\section{B. $p \bar{p} \rightarrow \mathcal{O} \rightarrow$ hadrons}

The coupling of $\mathcal{O}$ to $p \bar{p}$ is allowed by flavor $\mathrm{SU}(3)$. However, it is most likely small because so many channels are open aside from $p \bar{p}$ when $g g g$ of $\mathcal{O}$ turn in hadrons. Although accurate estimate of this coupling is not possible, we can make a reasonable guess with the charmonium decay branching into $p \bar{p}$.

The $J=1$ partial-wave cross section for $p \bar{p} \rightarrow \mathcal{O} \rightarrow$ all in the resonance region is given by the standard formula,

$$
\sigma_{r e s}(p \bar{p} \rightarrow \mathcal{O} \rightarrow a l l)=\frac{3 \pi}{|\mathbf{p}|^{2}} \frac{B_{i n} \Gamma_{\mathcal{O}}^{2}}{4\left(W-m_{\mathcal{O}}\right)^{2}+\Gamma_{\mathcal{O}}^{2}}
$$

where $W$ and $\mathbf{p}$ are the $\mathrm{cm}$ energy and momentum, and $B_{\text {in }}$ is the decay branching fraction for $\mathcal{O} \rightarrow p \bar{p}$. Since $J / \psi$ decays through $g g g$, a reasonable guess is to equate $B_{\text {in }}$ with $\mathrm{B}(J / \psi \rightarrow p \bar{p}) / \mathrm{B}(J / \psi \rightarrow g g g)=0.24 \%$. Then the peak cross section is

$$
\sigma_{\text {peak }}=3.5 \mu \mathrm{b}
$$

for $m_{\mathcal{O}} \simeq 3.7 \mathrm{GeV}$. Since the $p \bar{p}$ total cross section is approximately $60 \mathrm{mb}$ at this energy, there is no chance to see $\mathcal{O}$ as a resonance in $p \bar{p}$ annihilation.

Nonresonant production of $\mathcal{O}$ in conjunction with other light hadron(s), e.g., $p \bar{p} \rightarrow \mathcal{O} X$, is suppressed by the feeble coupling of $q \bar{q} \rightarrow g g g$ (the "disconnected quark diagram") for an identifiably narrow $\mathcal{O}$. This suppression is severe even for $\phi$, which is much lighter: $\sigma(\pi p \rightarrow$ $\phi X) / \sigma(\pi p \rightarrow \omega X) \approx 1 / 100$. Unlike $J / \psi$ search by $J / \psi \rightarrow \ell^{+} \ell^{-}$, absence of a discriminating decay characteristic will make it hard to identify $\mathcal{O}$.

\section{SIGNALS OF $\mathcal{O}-\psi^{\prime}$ MIXING IN $\psi^{\prime}$ DECAY}

The only chance to produce and directly observe the vector glueball $\mathcal{O}$ will be in the case that the decay width is very narrow. Our numerical estimate is based on the assumption that $\psi \rightarrow \mathcal{O} \rightarrow$ hadrons and $\psi^{\prime} \rightarrow$ ggg $\rightarrow$ hadrons add up without interference in the total rates. Most generally, $\mathcal{E}$ should be left as a parameter of $O(1)$. If the width $\Gamma_{\mathcal{O}}$ happens to be wider than a few $\mathrm{MeV}$ and the resonance search is not feasible, $\mathcal{O}$ must be searched by the indirect signatures in the $\psi^{\prime}$ decay that result from the $\mathcal{O}-\psi^{\prime}$ mixing. Let us explore such indirect signatures.

If $\psi^{\prime}$ is purely an $s$-wave bound state of $c \bar{c}$, and its hadronic decay occurs through the perturbative threegluon decay alone, we expect

$$
\begin{aligned}
\frac{\mathrm{B}\left(\psi^{\prime} \rightarrow g g g \rightarrow a l l\right)}{\mathrm{B}(J / \psi \rightarrow g g g \rightarrow a l l)} & \simeq\left(\frac{\alpha_{s}\left(m_{\psi^{\prime}}\right)}{\alpha_{s}\left(m_{J / \psi}\right)}\right)^{3} \frac{\mathrm{B}\left(\psi^{\prime} \rightarrow \ell^{+} \ell^{-}\right)}{\mathrm{B}\left(J / \psi \rightarrow \ell^{+} \ell^{-}\right)} \\
& =0.13 \pm 0.03
\end{aligned}
$$

The similarity of $\psi^{\prime}$ to $J / \psi$ led us to suspect that the same relation may hold approximately for individual exclusive channels as well. This naive rule of thumb was referred to as "the $14 \%$ rule". However, experiment has shown very large deviations from the "rule" for many decay modes 12. The ratio is most prominently off for $\rho \pi$, by a factor of 60 or more. This is the $\rho \pi$ puzzle.

\section{A. Even- $G$ channels}

The $\mathcal{O}-\psi^{\prime}$ mixing affects only the final states of $I=0$. Since $J^{P C}=1^{--}$, they are odd in G-parity. Since the even- $G$ states fed by $\psi^{\prime} \rightarrow \gamma^{*} \rightarrow$ hadrons are immune to the mixing, we should find

$$
\begin{aligned}
\frac{\mathrm{B}\left(\psi^{\prime} \rightarrow h(G=+)\right)}{\mathrm{B}(J / \psi \rightarrow h(G=+))} & \simeq \frac{\mathrm{B}\left(\psi^{\prime} \rightarrow \ell^{+} \ell^{-}\right)}{\mathrm{B}\left(J / \psi \rightarrow \ell^{+} \ell^{-}\right)} \\
& =0.15 \pm 0.03
\end{aligned}
$$

Among the decay modes of even $G$ so far analyzed,

$$
\frac{\mathrm{B}\left(\psi^{\prime} \rightarrow \omega \pi\right)}{\mathrm{B}(J / \psi \rightarrow \omega \pi)}=0.09 \pm 0.05
$$

which is off from Eq. (15), but only one standard deviation. A higher precision is desired for $\psi^{\prime} \rightarrow \omega \pi$. In comparison the $\rho \pi$ modes is severely suppressed as

$$
\frac{\mathrm{B}\left(\psi^{\prime} \rightarrow \rho \pi\right)}{\mathrm{B}(J / \psi \rightarrow \rho \pi)}<0.002
$$

The same ratio is $<0.021$ for $\omega \eta$ and $\rho a_{2}$, which are both odd in $G$. It is not true that all odd- $G$ channels are suppressed; the ratio is $0.18 \pm 0.05$ for $b_{1}^{ \pm} \pi^{\mp}$ over 0.13 of Eq. (14). The ratio is much larger for $\omega \eta^{\prime}(0.5 \pm 0.4)$, though the error is large.

There are a few other even- $G$ channels in the $J / \psi$ decay for which good measurements already exist. Among them are $\pi^{+} \pi^{-}, \rho \eta$, and $\rho \eta^{\prime}$. These decay modes should be measured carefully for $\psi^{\prime}$ for comparison. There is one $\psi^{\prime}$ decay mode that was observed with a large branching fraction but not yet identified in the $J / \psi$ decay. That is, $\psi^{\prime} \rightarrow \rho^{0} \pi^{+} \pi^{-}$with $\mathrm{B}\left(\psi^{\prime} \rightarrow \rho^{0} \pi^{+} \pi^{-}\right)=(3.7 \pm 0.6 \pm 0.9) \times$ $10^{-4}$ [5]. Only $\mathrm{B}\left(J / \psi \rightarrow \pi^{+} \pi^{-} \pi^{+} \pi^{-}\right)=(4.0 \pm 1.0) \times$ $10^{-3}$ [6] is quoted for $J / \psi$ [6]. If the $\mathcal{O}-\psi^{\prime}$ mixing is the solution to the puzzles, $\mathrm{B}\left(J / \psi \rightarrow \rho^{0} \pi^{+} \pi^{-}\right)$should obey Eq. (15) so that a large fraction of $J / \psi \rightarrow \pi^{+} \pi^{-} \pi^{+} \pi^{-}$ should consist of $J / \psi \rightarrow \rho^{0} \pi^{+} \pi^{-}$. No attempt has yet been made to search $a_{J} \pi(\mathrm{J}=0,1,2)$ in $\pi^{+} \pi^{-} \pi^{+} \pi^{-}$. It is worth efforts for testing of the $\mathcal{O}-\psi^{\prime}$ mixing.

\section{B. $\gamma h$ modes}

Some final states that consist of one photon and a hadron have large branching fractions. It is often interpreted that they arise from $J / \psi\left(\psi^{\prime}\right) \rightarrow \gamma g g \rightarrow \gamma h$ since the $\alpha$ suppression is partially compensated with lack of one power of $\alpha_{s}$ in $\gamma g g$. If this interpretation is right, the 
$\mathcal{O}-\psi^{\prime}$ mixing should little affect the $\gamma h$ modes. Then we would expect

$$
\frac{\mathrm{B}\left(\psi^{\prime} \rightarrow h \gamma\right)}{\mathrm{B}(J / \psi \rightarrow h \gamma)} \simeq \frac{\mathrm{B}\left(\psi^{\prime} \rightarrow \ell^{+} \ell^{-}\right)}{\mathrm{B}\left(J / \psi \rightarrow \ell^{+} \ell^{-}\right)} .
$$

At present, three decay modes are available for comparison: The ratios are $0.06 \pm 0.04$ for $\gamma \eta, 0.036 \pm 0.012$ for $\gamma \eta^{\prime}$, and $0.22 \pm 0.16$ for $\gamma f_{2}(1270)$. The errors are still large for $\gamma \eta$ and $\gamma f_{2}$. But the ratio for $\gamma \eta^{\prime}$ is clearly off the $\mathcal{O}-\psi^{\prime}$ mixing predicton, $0.15 \pm 0.03$. This may be taken against the $\mathcal{O}-\psi^{\prime}$ mixing. Or else the decay branching $\mathcal{O} \rightarrow \gamma \eta^{\prime}$ is enhanced for some reason. We recall that the $\eta^{\prime}$ yield tends to be larger than theoretical expectations in many other processes, e.g., $B$ decay. The common theme is the QCD anomalies involved in them. Together with higher precision in experiment, better theoretical understanding is needed for $\gamma h$, particularly for $\gamma \eta^{\prime}$.

\section{Alternatives to $\mathcal{O}-\psi^{\prime}$ mixing}

An alternative to the $\mathcal{O}-\psi^{\prime}$ mixing is to postulate that $\psi^{\prime}$ contains a large $d$-wave component of $c \bar{c}$, much larger than we have so far accepted. The $d$-wave content of $\psi^{\prime}$ was studied in the nonrelativistic model in the past 13,14. The amount of the $d$-wave appears to be considerably smaller than what is relevant to the issues in the $\psi^{\prime}$ decay. There is also an inconsistency with the radiative decays of $\psi^{\prime}$ [10]. However, these problems may well be due to the nonrelativistic potential model. Let us abandon the nonrelativistic model for the moment and assume in an ad hoc manner that a large $d$-wave component exists in $\psi^{\prime}$. In this case $\psi^{\prime} \rightarrow \ell^{+} \ell^{-}$would also be affected by the mixing, but differently from $\psi^{\prime} \rightarrow$ ggg $\rightarrow$ hadrons. Therefore, the branching fractions of even- $G$ channels would no longer obey the rule of Eq. (15) precisely.

One variation of this scenario is to replace the large $d$ wave component with the virtual $D \bar{D}$ configuration. In this case $D \bar{D} \rightarrow \ell^{+} \ell^{-}$is dynamically suppressed since both $c \bar{c}$ and $q \bar{q}(q=u, d)$ must be annihilated into $\gamma^{*}$ to create $\ell^{+} \ell^{-}$. In other words, the form factor suppression of $D \bar{D}$ occurs relative to the hard $c \bar{c}$ annihilation. Therefore $\psi^{\prime} \rightarrow \ell^{+} \ell^{-}$is reduced by the amount of the $D \bar{D}$ component. Then Eq. (14) would no longer hold. Although the hadrons from $\overline{D D}(D=c \bar{u}, c \bar{d})$ contains an $\mathrm{SU}(3)$-octet $I=0$ component $\left(\lambda_{8}\right)$ in addition to an $\mathrm{SU}(3)$ singlet, it will not be easy to identify this octet mixture, separating from the isoscalar electromagnetic contribution and the strong SU(3) breaking effects.

\section{SUMMARY AND REMARKS}

The chance of finding $\mathcal{O}$ exists only if its width is very narrow by the short-distance QCD suppression. Otherwise $\mathcal{O}$ will show up only in the $\psi^{\prime}$ decay indirectly. Even if the $\rho \pi$ puzzle is set aside, the proximity of the predicted $\mathcal{O}$ mass to the $\psi^{\prime}$ mass inevitably generates some $\mathcal{O}-\psi^{\prime}$ mixing. This will be the only experimental clue to the vector glueball of width wider than a few $\mathrm{MeV}$. It is therefore worth studying the mixing effect seriously.

\section{ACKNOWLEDGMENTS}

I am grateful to S.J. Brodsky, F.A. Harris, J.L. Rosner, and S.F. Tuan for useful conversations and/or communications on the $\rho \pi$ puzzle and related subjects during the past few years. Thanks are also due to P. Wang for informing me of the prospect of the Beijing charm experiment. This work was supported in part by the Director, Office of Science, Office of High Energy and Nuclear Physics, Division of High Energy Physics, of the U.S. Department of Energy under contract DE-AC03-76SF00098 and in part by the National Science Foundation under grant PHY-0098840.

[1] C.J. Morningstar and M.J. Peardon, Phys. Rev. D 60, 034509 (1999). For review, see also J. Kuti, heplat/9811021.

[2] T. Barnes et al., Nucl. Phys. B198, 380 (1982); C.E. Carlson et al., Phys. Rev. D 27, 1556 (1983).

[3] W-S. Hou et al, Phys. Rev. D 64, 014028 (2001).

[4] Mark II Collaboration, M.E.B. Franklin et al., Phys. Rev. Lett. 51, 963 (1983).

[5] F. A. Harris, hep-ex/9903036.

[6] Particle Data Group, D.E. Groom et al., Euro. Phys. J. C 15, 1 (2000).

[7] S.J. Brodsky, G.P. Lepage, and S.F. Tuan, Phys, Rev. Lett. 59, 621 (1987); S.J. Brodsky and M. Karliner, Phys. Rev. Lett. 78, 4682 (1997).

[8] M. Suzuki, Phys. Rev. D 63, 054021 (2001).

[9] Y.F. Gu and X.H. Li, Phys. Rev D 63, 114019 (2001).

[10] J.L. Rosner, Phys. Rev. D 64, 094002 (2001); hep$\mathrm{ph} / 0105327$.

[11] S.F. Tuan, Commun. Theor. Phys. 33, 285 (2000).

[12] The branching fractions of $\psi^{\prime}$ quoted here are mostly from Ref. [5]. Those which are not found in Ref. [- 5 ] have been taken from Ref.

[13] Y.P. Kuang and T.M. Yan, Phys. Rev. D 41, 155 (1990).

[14] J.L. Rosner, in Particles and Fields 3, Proc. 1988 Banff Summer Inst., Banff, Alberta, edited by A.N. Kamal and F.C. Khanna (World Scientific, Singapore, 1989), p.395. 